\title{
ATIVIDADES TEÓRICO-PRÁTICAS COM ÊNFASE EM FUNGOS: UMA PROPOSTA PARA O ENSINO MÉDIO
}

\section{THEORETICAL-PRACTICAL ACTIVITIES WITH EMPHASIS ON FUNGI: A PROPOSAL FOR MIDDLE SCHOOL}

\author{
Jeocarlas dos Santos Ferreira \\ Universidade Estadual do Piaui-UESPI / jeocarlasantos@gmail.com
}

Alexandre dos Santos Ferreira

Universidade Federal do Piaui-UFPI / alexandreflorests@gmail.com

\section{Resumo}

Devido à complexidade do conteúdo do Reino Fungi, estuda-los, exige estratégias de ação por parte dos professores. Com o objetivo de criar, aplicar e disponibilizar para os professores e alunos do ensino médio atividades que relacionam a teoria e a prática e que possibilitam uma melhor compreensão desse conteúdo em sala de aula. Este trabalho foi desenvolvido em uma turma de $2^{\circ}$ ano do ensino médio, de uma escola pública, localizada no município de Bom Jesus-PI. Nele apresenta uma proposta de atividade teórica com base nos resultados de uma análise do conteúdo de fungos exibido no livro didático adotado pela escola, e algumas atividades práticas com ênfase no filo Basidiomycota. Os resultados indicam que houve um maior esclarecimento aos alunos quando as informações contidas no livro didático foram repassadas atualizadas durante a aula teórica, e essa compreensão favoreceu a assimilação do conteúdo no decorrer das atividades práticas. Contudo, percebemos a importância de relacionar atividades teórico e práticas para obter um resultado mais satisfatório no processo de ensino-aprendizagem e que o professor precisa estar atento as informações apresentadas no livro didático, embora ele seja considerado uma ferramenta segura para o ensino, pode exibir informações errôneas e desatualizadas.

Palavras-chave: Fungos. Análise. Aulas práticas. Aula teórica. Livros didáticos. Ensino Médio

\section{Abstract}

Due to the complexity of the content of the realm of fungi, studying them, demands action strategies by teachers. With the aim of creating, applying and making available to teachers and students in Middle School activities that relate to theory and practice and that enable a better understanding of this content in the classroom. This work was developed in a secondary class of, Middle School of a public school, located in the municipality of Bom Jesus-Pli. It presents a proposal of theoretical activity based on the results of an analysis of the fungus content displayed in the textbook adopted by the school, and some practical activities with emphasis on the phylum Basidiomycota. The results indicate that students 
were given greater clarification when the information contained in the textbooks were updated during the theoretical lesson, and this understanding encouraged the assimilation of the content in the course of practical activities. However, we realise the importance of linking theoretical and practical activities to achieve a more satisfactory outcome in the teaching-learning process and that the teacher needs to be mindful of the information presented in the textbook, although he is considered a safe tool for teaching, can display erroneous and outdated information.

Keywords: Fungi. Analysis. Practical lessons. Theoretical lesson. Textbooks. Middle School

\section{Introdução}

De modo geral, os fungos são organismos eucarióticos, unicelulares ou pluricelulares, com micélio filamentoso composto por hifas septadas ou cenocíticas. A maioria das espécies apresenta parede celular composta por quitina, mas um pequeno grupo possui a predominância de celulose na sua composição. São heterotróficos, com nutrição por absorção auxiliada pela secreção de enzimas extracelulares, podendo ocorrer como parasitas ou sapróbios decompositores de uma grande variedade de substratos. A reprodução assexuada ocorre por meio de esporos, flagelados ou aflagelados, e a sexuada envolve a participação de gametas masculinos e femininos (MOORE-LANDECKER,1990).

Com base em estudos moleculares, químicos e morfológicos, nos últimos anos a sistemática dos fungos vem passando por uma série de mudanças e, atualmente, as espécies estão classificadas em três reinos: Protozoa, Chromista e Fungi. Destes, Fungi é o mais representativo compreendendo oito filos: Ascomycota, Basidiomycota, Chytridiomycota, Neocalimastigomycota, Blastocladiomycota, Glomeromycota, Microsporidia, Zygomycota e os fungos anamorfos (KIRK et al., 2008). Porém, como estes organismos apresentam características muito diversificadas, constantes alterações continuam sendo propostas, principalmente, em virtude de novas descobertas.

Desta forma, estudar fungos não tem sido uma tarefa fácil. $O$ fato de o grupo incluir organismos com uma sistemática tão variável e com caracteres morfológicos vegetativos e reprodutivos extremamente diversificados e complexos, a abordagem deste tema utilizando metodologia de ensino de forma exclusivamente teórica, com uso apenas do livro didático em sala de aula e com pouca experimentação prática, tornam o ensino deste grupo superficial (DIAS; SCHWARZ; VIEIRA, 2009).

O livro didático não pode continuar como única fonte de conhecimentos do professor, pois ele permanece aprisionado ao conteúdo apresentado e, no caso dos conteúdos de micologia, o mesmo acaba restringindo suas aulas somente a conceitos e termos presentes no livro. Outro agravante desta metodologia unicamente teórica está no fato de que, embora os livros didáticos passem por uma criteriosa análise, ainda são encontrados diversos exemplos de contradições entre as informações apresentadas no 
conteúdo. Assim, é papel do professor de Biologia encontrar e corrigir tais equívocos (VASCONCELOS; SOUTO, 2003), principalmente com apoio de atividades práticas.

Nos dias atuais é relevante que os professores utilizem metodologias diversificadas no intuito de despertar no aluno interesse e curiosidade, como por exemplo, atividades de campo e laboratório (MARTINS et al., 2010). Neste processo de aprendizagem, a teoria não deve estar desvinculada da prática, nem esta da teoria (GUEDES, 2009). No ensino de Biologia, as atividades práticas quando aliadas a teoria buscam e auxiliam a reconstrução de conceitos científicos significativos para o aluno. Em conjunto, as atividades teórico-práticas proporcionam condições para que haja o questionamento e reflexão na ação dos temas trabalhados, ou seja, a teoria se revisa na prática e a prática se enriquece na teorização (SOBRINHO, 2009).

Assim, o ensino de Biologia em sala de aula necessita de propostas inovadoras como meio alternativo ao padrão expositivo que se é visto em muitas escolas. Essas propostas podem ser uma aula de campo ou a construção de modelos biológicos em sala de aula (SILVA; BASTOS, 2012).

Devido à complexidade do conteúdo do Reino Fungi, estuda-los, exige estratégias de ação por parte dos professores, e uma dessa estratégias que o docente pode abordar dentro da sala de aula, são as aulas teóricas vinculadas as atividades práticas. Com o objetivo de criar, aplicar e disponibilizar para os professores e alunos do ensino médio atividades que relacionam a teoria e a prática e que possibilitam uma melhor compreensão desse conteúdo em sala de aula. O presente estudo apresenta uma proposta de atividade teórica com base nos resultados de uma análise do conteúdo de fungos apresentado no livro didático adotado pela escola e algumas atividades práticas com ênfase no filo Basidiomycota.

\section{Relações entre teoria e prática para o ensino de fungos}

No ensino de Biologia, Vendruscolo (2009) propõe que o professor necessita utilizar metodologias diferenciadas que chamem a atenção do aluno, motivando-o a estudar. Nesse contexto, para que os professores possam desenvolver um ensino eficiente voltados aos fungos, é importante que elaborem atividades práticas relacionando-as com a teoria, manifestando assim, a curiosidade dos educandos para que o conhecimento permaneça na sua memória.

Segundo Souza et al (2014), a prática e a teoria no cotidiano escolar devem receber a mesma dosagem de atenção. Embora as atividades práticas resultam na construção de momentos prazerosos no processo ensino-aprendizagem, estimulando o aluno a pensar e olhar em diferentes maneiras o assunto dado em sala de aula. A teoria é indissociável da prática; pois, toda teoria surge de uma prática (SOUZA et al, 2014).

A parte prática une-se com as informações teóricas e propiciam a construção de uma rede de esquemas mentais, auxiliando de um modo lógico e ordenado à compreensão dos conteúdos de fungos. Entretanto, uma atividade experimental precisa de muita atenção e concentração, pois envolve manipulação de materiais e instrumentos, conversa com os colegas, disponibilidade de equipamentos e movimentação constante (DEMO, 2002). 
Souza (2001), afirma que não se pode dar prioridade à teoria ou à prática, mas sim, fazer com que ambas se tornem recíprocas e constituam uma totalidade, produzida em um contexto, para que todo o potencial de ensino seja alcançado. Frente a isso, há necessidade de desconstrução do paradigma dicotômico entre o saber e o fazer, para que, então, apontem-se novos caminhos e perspectivas para as mudanças esperadas na formação profissional. Para Timóteo e Liberalino (2003), a teoria e a prática são complementares e não podem se dissociarem uma da outra na busca da construção do conhecimento. Entre as principais funções das aulas práticas estão: despertar e manter 0 interesse dos alunos, envolver os estudantes em investigações científicas, desenvolver a capacidade de resolver problemas e compreender conceitos básicos. Assim, as aulas práticas são importantes porque podem ser favoráveis na assimilação do conteúdo e observação dos elementos que foram expostos pelo professor na aula teórica (VENDRUSCOLO, 2009).

Segundo Pereira et al (2006), uma metodologia construtivista de ensino implica em repensar o papel da escola e do professor como orientadores da aprendizagem efetiva e significativa dos alunos, podendo contribuir decisivamente com a melhoria da qualidade do ensino da Biologia. Entretanto, o ensino de conceitos na aprendizagem significativa é um processo em que uma nova informação se encaixa com uma estrutura de conhecimento específica preexistente na estrutura cognitiva de quem aprende, ou seja, a essência do processo de aprendizagem significativa se baseia em ideias simbolicamente expressas que estejam relacionadas a algum aspecto relevante da estrutura do conhecimento (MOREIRA; MASINI, 2006)

O ensino do Reino Fungi na maioria das escolas é um ensino simplesmente tradicional, não possibilitando que o aluno tenha uma aprendizagem científica significativa e efetiva. É comum, os professores ficarem restritos somente ao livro didático, tornando-o a principal ferramenta de ensino para realizar as aproximações dos alunos com os seres vivos em geral (PEREIRA et al., 2006). O livro didático precisa ser complementado com o uso de métodos e materiais paradidático, pois ampliando-se as fontes de informação, ampliam-se também as possibilidades de aprendizagem.

É necessário, então, analisar se as metodologias utilizadas pelo professor estão trazendo resultados positivos. Afinal, os métodos adotados pelo docente precisam estar submetidos aos objetivos educacionais e vir ao encontro de um aprendizado expressivo para o aluno. É importante lembrar que somente algumas mudanças nas técnicas ou recursos utilizados não implicam em mudanças na abordagem do conteúdo (AMARAL, 2006). Cabe ressaltar, que utilizar metodologia de ensino que desperte no aluno o interesse pelo processo de construção do conhecimento é uma medida que pode permitir sucessos ou redirecionamentos para a prática docente (FAGUNDES; GONZALEZ, 2006).

Sendo assim, como relatado por Cunha (2001), para se ter uma boa relação com seu aluno é natural a preocupação com os métodos que serão utilizados para melhorar a aprendizagem. $O$ autor também enfatiza que o bom professor é aquele que não permanece somente a uma única metodologia, mas conforme as necessidades de aprendizagem de seus alunos elas são modificadas. É importante salientar que as práticas podem e devem ser utilizadas, mas não como único instrumento didático, e sim 
em sintonia com as demais estratégias de ensino (CAMPOS; NIGRO, 2009). Nesse caso, em consonâncias com as aulas teóricas.

\section{Desenvolvimento da pesquisa}

O presente trabalho foi desenvolvido em uma escola pública, situada na cidade de Bom Jesus-PI. A pesquisa abrangeu estudantes do segundo ano do ensino médio, em uma turma composta de 30 alunos.

\section{Planejamento e realização das atividades}

\section{Atividade Teórica}

Para a realização da atividade teórica, foi realizado uma análise do conteúdo de fungos. O conteúdo foi analisado tomando como base o critério sobre abordagem teórica utilizados por Silva e Junior (2016) em seu trabalho de análise sobre o conteúdo dos fungos nos livros didáticos indicados pelo PNLD 2012. Dentro desse critério foi observado se o livro didático apresentava de forma atualizada as características gerais, a estrutura e morfologia e a classificação bem como a evolução dos fungos.

A metodologia usada durante a aula teórica, foi a apresentação dos resultados da análise para a turma e o professor. O objetivo dessa aula consistiu em mostrar ao professor a importância de não basear suas aulas somente ao conteúdo presente no livro, mas buscar diferentes fontes de conhecimentos para que não ocorra o repasse de informações incompletas ou errôneas aos seus alunos.

\section{Atividades práticas}

Para a realização das atividades práticas, foram divididas em duas etapas: A primeira etapa foi realizada pelos autores, na qual elaboraram um manual com técnicas de coleta, preservação e herborização de basidiomicetos, a partir das recomendações de GIMENES (2010). Estas recomendações foram selecionadas e adaptadas à realidade da escola, possibilitando um material fácil e acessível aos alunos e professores. O conteúdo deste manual abordou os seguintes pontos:

1. Realização de coleta no campo, incluindo quais materiais são utilizados;

2. Principais características morfológicas importantes para identificação dos espécimes;

3. Herborização de material seco e preservação em álcool 70\%.

E, para finalizar esta primeira etapa, houve as coletas dos fungos. Estas coletas foram feitas preferencialmente durante o período chuvoso, percorrendo-se de forma aleatória, em diferentes áreas do município de Bom Jesus. Os espécimes de basidiomicetos coletados, foram fotografados e guardados em sacos individuais de papel. No laboratório do Campus Professora Cinobelina Elvas/UFPI, houve a identificação, baseadas nas seguintes bibliografias: Abrahão (2009); Putzke (2012); Neves et al (2013).

A segunda etapa das atividades práticas foi desenvolvida na escola. Após a elaboração do manual e o término das coletas, voltamos a escola. No início, organizamos 
a turma e distribuímos a ela e ao professor presente, os espécimes identificados e o manual previamente elaborado. Após, explicamos a turma sobre o conteúdo do manual e sua relação com os espécimes de fungos. Esperamos alguns minutos para que lessem o conteúdo. Depois, com a ajuda dos discentes, houve a montagem da minicoleção biológica. Durante esta aula prática procurou responder os seguintes questionamentos:

- O que são fungos?

- Quais as principais características dos fungos?

- Existem fungos comestíveis na minicoleção?

- Quais as partes morfológicas dos basidiomicetos podem ser utilizadas na para sua identificação?

Ainda durante a aula, os alunos construíram um modelo biológico de cogumelos. Por meio dessa atividade, buscamos associar e discutir as principais estruturas morfológicas destes fungos tratados no conteúdo teórico e na montagem da minicoleção. O material usado para a elaboração do modelo foram fitas adesivas, papeis coloridos, tintas, pinceis e isopor.

\section{Resultados e discussão}

A aula teórica foi ministrada no dia 29 de agosto de 2014. Nela, apresentamos aos alunos os seguintes resultados da análise. $\mathrm{Na}$ parte da sistemática apresentada, verificamos que estava desatualizada e, às informações sobre a morfologia como a produção de basidiósporos, presença do píleo ou chapéu, e de himênio, não foram explanadas como características típicas para identificação de um basidiomiceto. Os membros desse filo são diagnosticados pela célula sexual clavada denominada de basídio e que produz no seu ápice quatro prolongamentos que são os esterigmas, na extremidade dos quais se formam, em geral, quatro basidiósporos. O esporóforo é denominado basidioma, e pode apresentar uma grande diversidade de formas cores, características de grande importância para a identificação das espécies (NEVES et al,2013). Além dessas características microscópicas, são usadas também a descrição das estruturas macroscópicos nesse conjunto de caracteres para identificação e estudos dos táxons.

A compreensão dessas informações forneceu aos alunos um maior esclarecimento de como são feitas a classificação desses seres vivos, possibilitando a eles, um olhar mais criterioso ao se depararem com algum espécime no seu cotidiano. Para Santos et al (2007), o estudante tem seu caráter investigativo desenvolvido conforme a apresentação do conteúdo de ciência, presente nos livros. Este é um dos desafios que muitos professores enfrentam quando abordam conteúdos em suas aulas com base somente nos livros didáticos. Não é aconselhável o professor depender exclusivamente ou em excesso do livro didático para não se tornar refém do conteúdo presente (ou ausente) e dos erros e inadequações da obra (ROSA; MOHR,2010). É necessário, então, analisar se as metodologias utilizadas por ele estão trazendo resultados positivos. Afinal, os métodos adotados pelo docente precisam estar submetidos aos objetivos educacionais e vir ao encontro de um aprendizado expressivo para o aluno. É importante lembrar que somente algumas mudanças nas técnicas ou recursos utilizados não implicam em mudanças na 
abordagem do conteúdo (AMARAL, 2006). Trazer informações adicionais que não contém no livro didático não fogem do objetivo de uma aula teórica.

No que diz respeito à classificação do Reino Fungi, segundo o mais recente dicionário de fungos (Kirk et al, 2008), atualmente, existem oito filos. Contudo, o livro didático adotado na escola onde este estudo foi aplicado menciona a existência de apenas Ascomycota, Basidiomycota, Chytridiomycota e Zygomycota (GEWANDSZNAJDER, 2011). Ressaltando, que estes são os grupos mais representativos e comumente encontrados no dia-a-dia. Quanto à classificação das micorrizas, o livro não fez referência a nenhum grupo em que estão inseridos, ou seja, não houve a menção do filo Glomeromycota, no qual os representantes deste grupo de fungos estão atualmente incluídos. Foi bastante interessante a abordagem desse assunto durante a aula, justamente por mostrar aos alunos que a classificação dos fungos está em constante modificação. Pois, desde o início ela vem passando por tais modificações substanciais. Essas modificações na taxonomia do grupo são graças aos resultados de estudos de biologia molecular que são incorporados juntos com os dados obtidos dos estudos com sistema enzimático (COIMBRA,2013;SCHUBER et al,2012).Tal compreensão dos alunos sobre a classificação dos fungos, forneceu-os a entender como ocorreu à origem evolutiva, já que no livro apresentou evidências evolutivas que mostram que os fungos são mais relacionados aos animais do que às plantas, embora a maioria dos alunos associavam esses seres vivos como espécies do Reino Plantae. Então, foi esclarecido que no início, os mesmos não eram reconhecidos como um grupo distinto de organismos, porém, ligados às plantas, até que Whittaker (1969) propôs a criação de cinco reinos, dentre estes o Reino Fungi.

Segundo Pereira et al. (2006), uma metodologia construtivista de ensino implica em repensar o papel da escola e do professor como orientadores da aprendizagem efetiva e significativa dos alunos, podendo contribuir decisivamente com a melhoria da qualidade do ensino da Biologia. Entretanto, o ensino de conceitos na aprendizagem significativa é um processo em que uma nova informação se encaixa com uma estrutura de conhecimento específica preexistente na estrutura cognitiva de quem aprende, ou seja, a essência do processo de aprendizagem significativa se baseia em ideias simbolicamente expressas que estejam relacionadas a algum aspecto relevante da estrutura do conhecimento (MOREIRA; MASINI, 2006). Como o professor é o principal mediador do conhecimento, cabe a ele fazer essa relação entre aquilo que o aluno já conhece com 0 que ele precisa conhecer, e apresentando as correções necessárias. "Isto apoia o conceito de que quanto mais se relaciona o conteúdo de maneira substancial e não arbitrária com algum aspecto de estrutura cognitiva prévia que the for relevante, mais próximo se está da aprendizagem significativa (KIMURA et al,2013, p. 264)".

Com relação aos resultados das atividades práticas. Na primeira etapa, houve a coleta de 50 espécimes, mas somente 11 táxons de basidiomicetos (Quadro 1) foram identificados e usados para a montagem do pequeno herbário. Após a distribuição dos espécimes, como forma de envolver os alunos e estimula-los a participar do desenvolvimento da aula, foram feitos os questionamentos propostos na metodologia. Visto que é preciso a participação do aluno e não apenas em tê-lo como observador passivo (FERNANDES et al,2013). Quando perguntado: o que são fungos? E quais as 
principais características dos fungos? Observamos que a maioria dos estudantes apresentaram dificuldades em conceitua-los, mas afirmaram que não eram plantas, já que na aula teórica foi apresentado que estes seres vivos são diferentes das plantas. $\mathrm{Na}$ ocasião, também foi possível discutir na prática a existência dos grupos de classificação, de acordo com as suas características observadas.

Assim, as aulas práticas são importantes porque podem ser favoráveis na assimilação do conteúdo e observação dos elementos que foram expostos pelo professor na aula teórica (VENDRUSCOLO, 2009). Essa assimilação entre aula teórica e aula prática, só é possível por meio de planejamento e um maior embasamento do conteúdo por parte do professor. Pois é por meio de uma aula teórica contextualizada com uma atividade prática que os alunos conseguem compreender certos conceitos e características.

Quadro 01 - Relação de táxons de basidiomicetos coletados e utilizados na montagem da minicoleção biológica.

\begin{tabular}{|c|c|c|c|}
\hline Reino Fungi & \multicolumn{3}{|c|}{ Substratos } \\
\hline $\begin{array}{c}\text { Filo } \\
\text { Basidiomycota }\end{array}$ & Estrume & Folhedos & Troncos \\
\hline \multicolumn{4}{|l|}{$\begin{array}{c}\text { Ordem } \\
\text { Agaricales }\end{array}$} \\
\hline $\begin{array}{l}\text { Agrocybe } \\
\text { retigera }\end{array}$ & * & & \\
\hline $\begin{array}{l}\text { Leucocoprinus } \\
\text { birnbaumi }\end{array}$ & & & * \\
\hline $\begin{array}{l}\text { Macrolepiota } \\
\text { sp. } 1\end{array}$ & & * & \\
\hline $\begin{array}{l}\text { Macrolepiota } \\
\text { sp. } 2\end{array}$ & & * & \\
\hline Marasmius sp & & * & \\
\hline \multicolumn{4}{|l|}{$\begin{array}{c}\text { Ordem } \\
\text { Aphyllophorales }\end{array}$} \\
\hline $\begin{array}{l}\text { Ganoderma } \\
\text { resinaceum }\end{array}$ & & & * \\
\hline $\begin{array}{l}\text { Lentinus } \\
\text { Crinitus }\end{array}$ & & & * \\
\hline Lentinus sp & & & * \\
\hline $\begin{array}{l}\text { Pycnoporus } \\
\text { sanguineus }\end{array}$ & & & * \\
\hline $\begin{array}{c}\text { Trichaptum } \\
\text { perrottetii }\end{array}$ & & & * \\
\hline $\begin{array}{l}\text { Schizophyllum } \\
\text { sp }\end{array}$ & & & * \\
\hline
\end{tabular}

Em outra questão, sobre a existência de fungos comestíveis na minicoleção, notamos que os alunos no sentido de elaborar as suas respostas sobre os questionamentos realizados trabalhavam com a observação e a discussão de como reconhecer um cogumelo venenoso e separá-lo dos comestíveis. Embora seja uma 
indagação de difícil resposta, pois não existe uma característica única e facilmente visível que permita essa separação, os alunos mantiveram ativos na busca por eventuais respostas, alguns por exemplo, utilizaram os recursos da internet para encontrar uma solução. Percebe-se que o uso de atividades práticas propostas como problemas a serem resolvidos permiti aos alunos realizarem um conjunto de observações, tarefas de classificações, entre outras, cabendo, ao docente, um papel de orientador da aprendizagem (CAMPANÁRIO; MOYA, 1999). O professor deve orientar durante uma aula prática um direcionamento diante dos questionamentos e soluções propostos pelos alunos para que ocorra um resultado satisfatório e assertivo. Alguns alunos acreditavam que a presença do anel no estipe, ou o tamanho do píleo são indícios de cogumelos venenosos. No entanto, Isso não é verdade, uma vez que são necessárias uma série de características associadas para a identificação de espécies venenosas e comestíveis (MOURA,2008). Nesse sentido, os alunos puderam compreender o porquê de não ingerir qualquer cogumelo encontrado na natureza, e que os cogumelos utilizados na alimentação humana só podem ser adquiridos de fontes confiáveis.

Nessa aula também foi possível discutir sobre os valores nutricionais dos cogumelos comestíveis, algo que era desconhecido pela maioria dos estudantes. Pois, segundo Orsine, Brito e Novaes (2012), os cogumelos podem ser acrescidos aos hábitos alimentares normais da população por ser alimentos com excelentes características nutricionais, como alto teor de proteínas, fibras alimentares, minerais, vitaminas, diversas substâncias bioativas com propriedades farmacológicas e baixo teor de lipídeos. Dessa maneira o aluno passa a perceber que os fungos têm grande importância não só na natureza, mas também em seu cotidiano e com aplicações em diversos setores da sociedade (SILVA; JUNIOR,2016).

Após o término da montagem da minicoleção biológica, os alunos foram conduzidos a criarem um modelo biológico de cogumelos utilizando materiais como garrafa pet, tesoura, cds velhos, isopor e fita adesiva. O uso de modelos didáticos gera maior compreensão do conteúdo teórico, proposto em sala de aula e, é um mecanismo alternativo para uma melhor assimilação por parte dos educandos (ROCHA et al, 2010). Através dessa atividade, os alunos conseguiram reproduzir na prática as características estruturais de um cogumelo, favorecendo o despertar de sua criatividade e uma maior interação entre os seus colegas dentro da sala de aula.

Esta atividade experimental foi uma estratégia didática que auxiliou na compreensão dos conhecimentos teóricos, em que os alunos diante de observações e procedimentos, alcançaram a aprendizagem. Segundo Rui (2013), realizando também aulas práticas com fungos, concluiu que essas atividades tratavam-se de uma oportunidade para os alunos fazerem comparações que coloquem em evidência similaridades e diferenças do grupo de organismos a ser estudado e se sensibilizem diante das diversidades de representantes que estão inseridos no cotidiano da sua vida.

Todo o material herborizado, juntamente com o modelo biológico, foi mantido na escola para uso dos professores e alunos. 


\section{Considerações finais}

Embora esse tipo de aula exija do professor maior tempo e preparo. Percebemos no decorrer desse estudo que relacionar atividades teórico e práticas favorece para os professores e alunos um resultado mais satisfatório no processo de ensino-aprendizagem. Isso porque durante as aulas houve uma motivação maior dos estudantes em participar das discussões propostas e uma compreensão dos mesmos em relação ao conteúdo de fungos. Pois este conteúdo é considerado um desafio para os professores da educação básica (SIMAS; FORTE,2013), justamente por ser analisado como um conteúdo bastante complexo e de difícil compreensão por parte dos alunos.

Os resultados desse trabalho também indicam que o professor precisa estar atento as informações apresentadas no livro didático, embora ele seja considerado uma ferramenta segura para o ensino, pode exibir informações errôneas e desatualizadas sobre um determinado assunto, nesse caso o conteúdo de fungos e comprometer sua abordagem no decorrer da aula teórica. Resultados de estudos também tem mostrado que, apesar dos livros didáticos disponibilizados pelo Ministério da Educação serem antes avaliados por equipes capacitadas, ainda há deficiências nas obras selecionadas (ESPÍNOLA, 2007; SILVA; JUNIOR,2016; ROSA; MORH, 2010). Cabe então ao professor evitar as abordagens incorretas do mesmo e corrigindo-as quando necessário com os seus alunos.

\section{Referências}

ABRAHÃO, M. C. Estudo da diversidade de basidiomicetos poliporóides de fragmentos florestais remanescentes do noroeste paulista. 2009. 240 f. Dissertação (Microbiologia) - Instituto de Biociências, Letras e Ciências Exatas da Universidade Estadual Paulista, São José do Rio Preto- SP.

AMARAL, I. A. Metodologia do Ensino de Ciências como produção social. Campinas: Faculdade de Educação, 2006. Disponível em: < http://www.fe.unicamp.br/ensino/graduacao/downloads/proesfMetodologiaEnsinoCienciasIvan.pdf. > Acesso em: 17 jul. 2014.

CAMPANÁRIO, J. M.; MOYA, A. ¿Cómo enseñar ciencias? Principales tendencias y propuestas. Enseñanza de las Ciencias, v. 17, n. 2, p. 179-192, 1999.

CAMPOS, M. C. C.; NIGRO, R. G. Teoria e Prática em Ciências na escola: o ensinoaprendizagem como investigação. 1ª ed. São Paulo: FTD, 2009.

COIMBRA, M. R.V. Fungos agaricóides (agaricales, Basidiomycota) da reserva biológica saltinho, Pernambuco: diversidade e aspectos moleculares. 2013. $79 f$. Dissertação (Centro de Ciências Biológicas)- Universidade Federal de Pernambuco, Recife-PE.

CUNHA, M. I. O bom professor e sua prática. São Paulo: Campinas, 2001.

DEMO, Pedro. Educar pela pesquisa. São Paulo: Autores Associados, 2002. 
DIAS, J. M. C; SCHWARZ, E. A; VIEIRA, E. R. A Botânica além da sala de aula, 2009. Disponível em: <http//www.diaadiaeducação.pv.gov/portals/pde/arquivos/ 8934.pdf? PDHPSESSID=2009043015481320 > Acesso em: 15 jul. 2014.

ESPÍNOLA, C. R. R. Aves na Escola: análise de livros didáticos do ensino fundamental. 2007. 63f. Monografia (Graduação em Ciências Biológicas) - Centro de Ciências Biológicas, Universidade Federal de Santa Catarina, Florianópolis, 2007.

FAGUNDES, J. A; GONZALEZ, C. E. F. Herbário escolar: suas contribuições ao estudo da Botânica no Ensino Médio. Paraná, 2006. Dissertação (Mestrado em tecnologia) - Universidade Tecnológica Federal do Paraná.

FERNANDES,M. C; SANTOS, F.L; PAULA, M. K; VIGÁRIO, F.A. Sequência didática para Ensinar Biologia - compreendendo os Fungos. Enciclopédia Biosfera, v.9, n.16, p. 23852392, 2013.

GEWANDESZNAJDER, F; LINHARES, S. Biologia hoje. $1^{\text {a }}$ edição. São Paulo: ática, 2011. cap. 5: Fungos, p. 82-90.

GIMENES, J. L. Fungos basidiomicetos: Técnicas de coleta, isolamento e subsídios para processos biotecnológicos. São Paulo, 2010. Disponível em: <http://www.biodiversidade.pgibt.ibot.sp.gov.br/Web/pdf/Fungos_Basidiomicetos_Luciana _J_Gimenes.pdf> Acesso em: 15 jul .2014.

GUEDES, S. T. R. A relação teoria e prática no estágio supervisionado. In: IX CONGRESSO NACIONAL DE EDUCAÇÃO - EDUCERE: POLÍTICAS E PRÁTICAS EDUCATIVAS DESAFIOS DA APRENDIZAGEM. 2009, Curitiba. Anais...Curitiba: Pontifícia Universidade Católica do Paraná, 2009. p. 11.

KIMURA, H. A; OLIVEIRA, S. G; SCANDORIEIRO, S; SOUZA, C.P; SCHURUFF, A.P; MEDEIROS, P. L; BODMAR, C.G ;SARMIENTO, P. J J;GAZAL, S. E .L;SANTOS, C. M. P ;KOGA, L.V;CYOIA, S.P NISHIO, K. E ;MOREY, T.A;TATIBANA, T. B; NAKAZATO, G; KOBAYASHI, T .K .R. Microbiologia para o Ensino Médio e Técnico: contribuição da extensão ao ensino e aplicação da ciência. Revista Conexão UEPG, v. 9, n.2, p. 264, 2013.

KIRK, P. M; CANON, P. F; MINTER, D. W; STALPERS, J. A. Dictionary of the fungi. CAB International, Wallingford, 2008.

MARTINS, E. K.; NOGUEIRA, M. K. F. S.; FERREIRA, A. R.; MORALES, A. G. M., A utilização de material didático botânico no ensino de ciências. In: II SIMPÓSIO NACIONAL DE ENSINO DE CIÊNCIA E TECNOLOGIA, n.157.,2010, Paraná. Resumos...Paraná: Universidade Tecnológica Federal do Paraná, 2010. p.14.

MOORE-LANDECKER, E. Fundamentals of the Fungi. $4^{\text {a }}$ ed. Prentice Hall, Inc.: New Jersey, 1996.

MOREIRA, M. A; MASINI, E. F. S. A teoria cognitiva da aprendizagem. In: Aprendizagem significativa: a teoria de David Ausubel. Editora Centauro. $2^{\mathrm{a}}$ ed. São Paulo. 2006. p. 17-33. 
MOURA, C.L.P. Determinação de elementos essenciais e tóxicos em cogumelos comestíveis por Análise por ativação com nêutrons. 2008. 117 f. DissertaçãoAutarquia Associada à Universidade de São Paulo, São Paulo-SP

NEVES. A. M; BASEIA, G.I; SANTOS, D. R. E; NETO, G. A. Guide to the Common Fungi of the Semiarid Region of Brazil. 1를 edição. Florianópolis: Tecc, 2013, p. 129.

ORSINE,C. V.J; BRITO, M. L; NOVAES, G.C. R.M. Cogumelos comestíveis: uso, conservação, características nutricionais e farmacológicas. Revista HCPA, v .32, n. 4, p. 452-460, 2012.

PEREIRA, M. G; GOLVEIA, Z. M. M; OLIVEIRA, G. L. C; PESSOA, M. C. R. A instrumentação do ensino de biologia através de materiais botânicos e suas implicações no processo de ensino e aprendizagem. 2006. Disponível em: <www.prac.ufpb.br > Acesso em: 15 jul. 2014.

PUTZKE, J. Cogumelos no sul do Brasil. $1^{\text {a }}$ edição, 2012. Disponível em: <http://www.bibvirt.futuro.usp.br/index.html>. Acesso em: 27 dez. 2014.

ROCHA, R. A; MELLO, N. W; BURITY, F. H. C. A utilização de modelos didáticos no ensino médio: uma abordagem em artrópodes. Saúde \& Ambiente, v.5, n.1, p.15-20, 2010.

ROSA, M. A.; MOHR, A. Os fungos na escola: análise dos conteúdos de micologia em livros didáticos do ensino fundamental de Florianópolis. Experiências em Ensino de Ciências, Cuiabá, v. 5, n. 3, p. 95-102, 2010

RUI, H. M. G. Atividades investigativas no ensino de ciências: Uma sequência didática sobre o tema fungos para o Ensino Fundamental.2013.179 f. Dissertação (Educação, em Ciências e Matemática) - Instituto Federal do Espírito Santo, Vitoria-ES.

SANTOS, J.C.; ALVES, L.F.A.; CORRÊA, J.J.; SILVA, E.R.L. Análise comparativa do conteúdo filo Mollusca em livro didático e apostilas do ensino médio de Cascavel, Paraná. Ciência \& Educação, v. 13, n.3, p. 311-322,2007.

SCHUBER, L.C.L;CRUZ, R.C.M; BONFIM, B.M.T;BRAND,D; PASTORE,M.G;ALMEIDA, M.M. Isolamento e seleção de fungos produtores de B-galactosidase. Evidência, v. 12 n. 1, p. 19-40, 2012.

SILVA, C. A; JUNIOR, M. N. Análise do conteúdo de fungos nos livros didáticos de biologia do ensino médio indicados pelo programa nacional do livro didático 2012. Revista Ciências e Ideias, v.7, n.3, p. 49-79,2016.

SILVA, M. S.; BASTOS, S. N. D. Ensino de microbiologia: percepção de docentes e discentes nas escolas públicas de Mosqueiro, Belém, Pará. In: ENCONTRO NACIONAL DE ENSINO DE CIÊNCIAS DA SAÚDE E DO AMBIENTE, 3, 2012, Niterói. Anais... Niterói: UFF, 2012. Disponível em: <http://www. ensinosaudeambiente.com.br/eneciencias/anaisiiieneciencias/trabalhos/T114.pdf> Acesso em: 15 jul. 2014.

SIMAS, E. S.; FORTES, S. T. Trilhando o mundo dos fungos: jogo didático para o ensino médio. In: CONGRESSO BRASILEIRO DE MICOLOGIA, 7., 2013, Belém. Resumos...Belém: Sociedade Brasileira de Micologia, 2013. p. 36. Disponível em: < 
http://www.micologia2013.com.br/LIVRO_DE_RESUMOS_DO_VII_CONGRESSOBRASILEIRO_DE_MICOLOGIA.pdf>. Acesso em: 4 jun. 2017.

SOBRINHO, R. S. A importância do ensino da biologia para o cotidiano. Fortaleza, 2009. 40 p. Monografia (Graduação em Ciências Biológicas) - Faculdade integrada da grande Fortaleza.

SOUZA, A.P.A; SILVA, R.J; ARRUDA, M. R; ALMEIDA, V.M.I.L; CARVALHO, T.E. A Necessidade da Relação Entre Teoria e Prática no Ensino de Ciências Naturais. UNOPAR Cient., Ciênc. Human. Educ, v. 15, n.esp, p. 395-401, 2014.

SOUZA, N. A. A relação teoria-prática na formação do educador. Semina: Ciencia. Sociologia. Humana, v. 22, p. 5-12, 2001.

TIMOTEO, R. P. S; LIBERALINO, F. N. Reflexões acerca do fazer pedagógico a partir de referências e diretrizes educacionais para a formação em enfermagem. Revista Brasileira de Enfermagem, v. 56, n. 4, p. 358-360, 2003.

VASCONCELOS, S. D; SOUTO, E. O livro didático de ciências no ensino fundamental proposta de critérios para análise do conteúdo zoológico. Revista Ciência \& Educação, v. 9, n. 1, p. 93-104, 2003.

VENDRUSCOLO, G. S. Botânica do ensino médio: uma análise metodológica com ênfase nas aulas práticas. Santa Catarina, 2009. p. 40. Monografia (Graduação em Ciências Biológicas) - Universidade Comunitária da Região de Chapecó.

WHITTAKER, R. H. New concepts of kingdoms of organisms. Science, v. 163, p.150-160, 1969.

Submissão: 11/10/2016

Aceite: 01/06/2017 